\title{
Design considerations for a laser-plasma linear collider
}

\author{
C. B. Schroeder, E. Esarey, C. G. R. Geddes, Cs. Tóth and W. P. Leemans \\ Lawrence Berkeley National Laboratory, Berkeley, California 94720, USA
}

\begin{abstract}
Design considerations for a next-generation electron-positron linear collider based on laser-plasma-accelerators are discussed. Several of the advantages and challenges of laser-plasmabased accelerator technology are addressed. An example of the parameters for a $1 \mathrm{TeV}$ laser-plasmabased collider is presented.
\end{abstract}

Keywords: laser-plasma accelerator, linear collider.

\section{INTRODUCTION}

Advanced acceleration techniques are actively being pursued to expand the energy frontier of future colliders. Although the exact minimum interesting energy of the next lepton collider will be determined by the Large Hadron Collider experiments that are presently underway, it is anticipated that $\geq 1 \mathrm{TeV}$ center-of-mass energy will be required. This energy is already near the limit of what can be constructed using conventional accelerator technology, given reasonable space and cost restrictions [1].

Laser-plasma accelerators [2] have demonstrated accelerating gradients on the order of $100 \mathrm{GV} / \mathrm{m}$, several orders of magnitude larger than conventional accelerators, which are limited to $\lesssim 100 \mathrm{MV} / \mathrm{m}$ by material break-down. Hence, employing laser-plasmaaccelerator technology has the potential to significantly reduce the main linac length (and, therefore, the cost) of a future lepton collider. Recent progress in the field of laserplasma accelerators, and in particular the demonstration of high-quality $\mathrm{GeV}$ electron beams at Lawrence Berkeley National Laboratory (LBNL) [3, 4], has increased interest in laser-plasma acceleration as a path toward a compact TeV-class linear collider.

\section{LASER-PLASMA ACCELERATION}

The amplitude of the accelerating field of a plasma wave driven by a resonant laser (pulse duration on the order of the plasma period) is approximately $E_{z} \approx\left(a^{2} / 2 \gamma_{\perp}\right) E_{0}$, where $a^{2}=7.3 \times 10^{-19}(\lambda[\mu \mathrm{m}])^{2} I_{0}\left[\mathrm{~W} / \mathrm{cm}^{2}\right]$ the normalized laser intensity, $\gamma_{\perp}=(1+$ $\left.a^{2} / 2\right)^{1 / 2}$ is the Lorentz factor associated with the quiver motion of the electrons in the laser field, and $E_{0}=m c \omega_{p} / e \simeq(96 \mathrm{~V} / \mathrm{m})\left(n_{0}\left[\mathrm{~cm}^{-3}\right]\right)^{1 / 2}$ is the characteristic plasma wave accelerating field amplitude, with $\omega_{p}=\left(4 \pi n_{0} e^{2} / m\right)^{1 / 2}$ the plasma frequency and $n_{0}$ the plasma number density. The quasi-linear regime is accessible for parameters such that $a^{2} / \gamma_{\perp}<k_{p}^{2} r_{L}^{2}$, where $r_{L}$ is the characteristic scale length of the transverse laser intensity. The transverse focusing force in the quasi-linear regime scales as $F_{\perp} \propto$ 


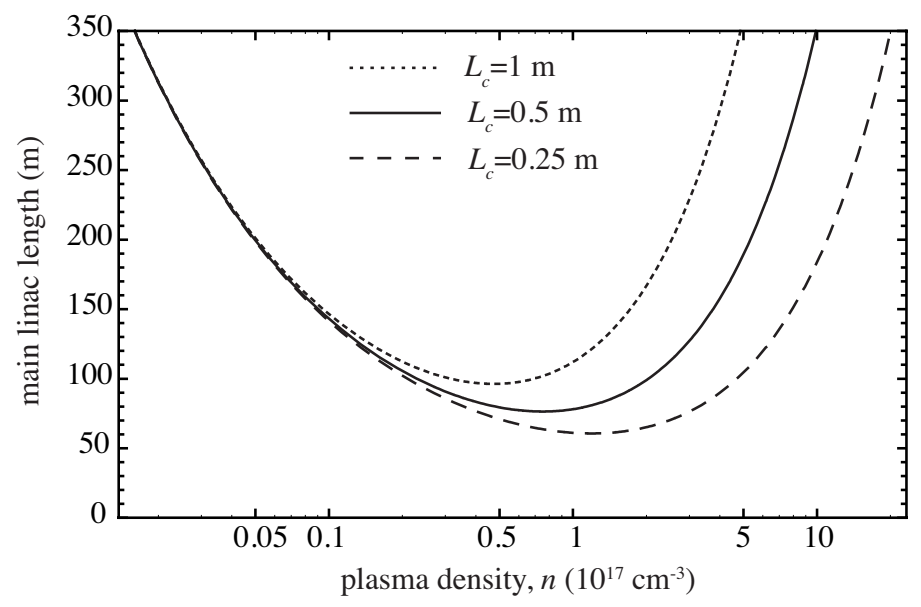

FIGURE 1. Main single-linac length versus plasma density $n_{0}$ for several laser in-coupling distances $L_{c}$, for $E_{b}=0.5 \mathrm{TeV}$ and $a_{0}=1.5$.

$k_{p}^{-1} \nabla_{\perp} a^{2}$, and, therefore, by shaping the transverse profile of the laser, the transverse forces in the accelerator can be controlled. Control over the focusing forces enables control of the beam dynamics (e.g., the beam matching condition). This control is not available in the highly-nonlinear blow-out (or bubble) regime, where the transverse forces are determined solely by the plasma density.

In the quasi-linear regime, the accelerating and focusing phase regions for electrons and positrons are nearly symmetric since the wakefield is nearly sinusoidal. In the blowout regime the accelerating and focusing region for positrons is severly reduced since the wakefield is highly nonlinear.

In general, the energy gain in a single laser-plasma accelerator stage may be limited by laser diffraction effects, dephasing of the electrons with respect to the accelerating field phase velocity (approximately the laser driver group velocity), and laser energy depletion into the plasma wave. Laser diffraction effects can be mitigated by use of a plasma channel (transverse plasma density tailoring), guiding the laser over many Rayleigh ranges $[3,5]$. Dephasing can be mitigated by plasma density tapering (longitudinal plasma density tailoring), which can maintain the position of the electron beam at a given phase of the plasma wave [6]. Hence the single-stage energy gain is ultimately determined by laser energy depletion. The energy depletion length scales as $L_{d} \propto n_{0}^{-3 / 2}$, and the energy gain in a single stage scales as with plasma density as $W_{\text {stage }} \propto n_{0}^{-1}$.

After a single laser-plasma accelerating stage, the laser energy is depleted and a fresh laser pulse must be coupled into the plasma for further acceleration. This coupling distance is critical to determining the overall accelerator length (average gradient of the main linac) and the optimal plasma density at which to operate. One advantage of laser-driven plasma acceleration is the potential for a short coupling distance between stages, and, therefore, the possibility of a high average accelerating gradient and a relatively short main linac length. The overall linac length will be given by $L_{\text {total }}=$ $\left[L_{\text {stage }}+L_{c}\right] E_{b} / W_{\text {stage }}$, where $L_{c}$ is the required coupling distance for a new drive laser (and space for any required beam transport and diagnostics), $E_{b}$ is the beam energy 
before collision, and $L_{\text {stage }} \approx L_{d}$ is the single-stage plasma length. Figure 1 plots the main linac length versus plasma density for several coupling distances, with $E_{b}=0.5 \mathrm{TeV}$ and $a_{0}=1.5$. Here the single-stage length and energy gain was calculated using a fluid code [7] to model the laser-plasma interaction. Plasma mirrors show great promise as optics to direct high-intensity laser pulses, requiring only tens of $\mathrm{cm}$ to couple a drive laser into a plasma accelerator stage [8].

\section{GENERAL COLLIDER DESIGN CONSIDERATIONS}

The rate of events in a collider is determined by the product of the collision cross section and luminosity. The geometric luminosity is

$$
\mathscr{L}=\frac{f N^{2}}{4 \pi \sigma_{x} \sigma_{y}}=\frac{P_{b}}{4 \pi E_{b}} \frac{N}{\sigma_{x} \sigma_{y}},
$$

where $f$ is the collision frequency, $N=N_{e^{-}}=N_{e^{+}}$is the number of particles per bunch (we assume equal number of particles per bunch for both electrons and positrons), $\sigma_{x}$ and $\sigma_{y}$ are the horizontal and vertical $\mathrm{rms}$ beam sizes, respectively, at the interaction point (IP), $E_{\mathrm{cm}}=2 \gamma m c^{2}=2 E_{b}$ is the center of mass energy, and $P_{b}=f N E_{b}$ is power in one beam. Since the cross section for electron-positron collisions scales as the inverse of the square of the center-of-mass energy, $\propto E_{\mathrm{cm}}^{-2}$, the luminosity must increase proportionally to maintain the collision rate. The luminosity requirement is approximately $\mathscr{L}\left[10^{34} \mathrm{~cm}^{-2} \mathrm{~s}^{-1}\right] \approx E_{\mathrm{cm}}^{2}[\mathrm{TeV}]$. Equation (1) indicates, for fixed beam power, the transverse beam density at the IP must be increased as the center-of-mass energy increases.

There are several limitations to the achievable beam density at IP. For example, these include the achievable beam emittance (given limitations on initial emittance and cooling methods), radiation effects during the final focus to the IP (Oide limit [9]), emittance growth in main linacs, and beam-beam interactions at the collision. Below we will examine the beam-beam interaction at the IP, as it dictates the the need for ultra-short bunches. Ultra-short bunches are intrinsically generated using plasma-based accelerators, allowing suppression of radiation generated by the beam-beam interaction. We will also examine an emittance growth mechanism unique to plasma-based accelerators, namely emittance growth due to Coulombic scattering of the beam with background plasma ions.

\section{Quantum beamstrahlung regime}

The beam-beam interaction at the IP produces radiation (beamstrahlung) that generates background for the detectors and increases the beam energy spread (resulting in loss of measurement precision). The beam-beam interaction is characterized by the Lorentz-invariant beamstrahlung parameter (mean field strength in the beam rest frame 

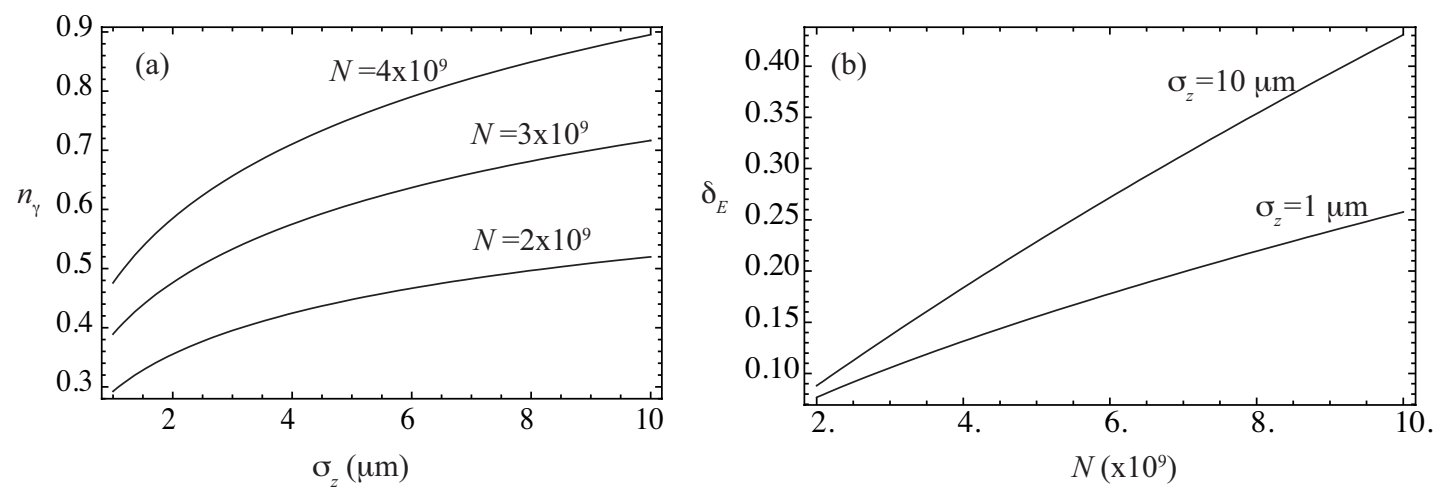

FIGURE 2. (a) Beamstrahlung photons emitted per electron $n_{\gamma}$ versus bunch length $\sigma_{z}$ and $N$. (b) Beamstrahlung induced energy spread $\delta_{E}$ versus $N$ and $\sigma_{z}$

normalized to the Schwinger critical field) [10]:

$$
\Upsilon \simeq \frac{5 r_{e}^{2} \gamma N}{6 \alpha \sigma_{z}\left(\sigma_{x}+\sigma_{y}\right)}=\frac{5 \sqrt{2 \pi} r_{e}^{2}}{12 \alpha m c^{2}}\left(\frac{E_{\mathrm{cm}}^{3} \mathscr{L}}{P_{b}}\right)^{1 / 2}\left(\frac{\sqrt{R}}{1+R}\right) \frac{N^{1 / 2}}{\sigma_{z}}
$$

where $r_{e}=e^{2} / m c^{2}, \alpha$ is the fine structure constant, $\sigma_{z}$ is the bunch length, and $R=\sigma_{x} / \sigma_{y}$ the aspect ratio of the beam at IP. As Eq. (2) indicates, using flat beams $R \ll 1$ reduces the beamstrahlung. In terms of the beamstrahlung parameter, the average number of emitted photons per electron is $n_{\gamma} \simeq 2.54\left(\alpha^{2} \sigma_{z} / r_{e} \gamma\right) \Upsilon\left(1+\Upsilon^{2 / 3}\right)^{-1 / 2}$ and the relative energy spread induced is $\delta_{E} \simeq 1.24\left(\alpha^{2} \sigma_{z} / r_{e} \gamma\right) \Upsilon^{2}\left(1+3 \Upsilon^{2 / 3} / 2\right)^{-2}$.

The current generation of linear colliders designs based on conventional technology operate in the classical beamstrahlung regime $\Upsilon \ll 1$. The next generation linear colliders with $E_{\mathrm{cm}} \gtrsim 1 \mathrm{TeV}$, will most likely operate in the quantum beamstrahlung regime with $\Upsilon \gg 1$. In the quantum beamstrahlung regime, assuming $E_{\mathrm{cm}}, P_{b}, \mathscr{L}$, and $R$ are fixed, the number of beamstrahlung photons scale as $n_{\gamma} \propto\left(N \sigma_{z}\right)^{1 / 3}$ and the induced beam energy spread scales as $\delta_{E} \propto\left(N \sigma_{z}\right)^{1 / 3}$. In this regime, beamstrahlung is reduced by using shorter bunches and smaller charge per bunch. Of course, reduction in charge per bunch is limited by luminosity requirements (i.e., if the bunch number decreases, then $f$ must be increased or the beam transverse dimensions decreased). For fixed beamstrahlung $n_{\gamma}\left(\right.$ and $\delta_{E}$ ), the luminosity per beam power scales as $\mathscr{L} / P_{b} \propto \sigma_{z}{ }^{-1 / 2}$, indicating short bunches are critical for next-generation linear colliders.

Figure 2(a) shows $n_{\gamma}$ versus $\sigma_{z}$ for several $N$, and Fig. 2(b) shows $\delta_{\gamma}$ in $N-\sigma_{z}$ parameter space. Unless otherwise noted the parameters of Table 1 were assumed. In general, the background must be $n_{\gamma}<1$, and $\delta_{E}$ a few tens of percent. For a $1 \mathrm{TeV}$ collider, micron bunch lengths are desirable using bunches with a few $10^{9}$ particles.

Plasma-based accelerators are intrinsically sources of ultra-short bunches since the scale length of the accelerating bucket in a plasma-based accelerator is the plasma wavelength $\lambda_{p}[\mu \mathrm{m}] \simeq 33 / \sqrt{n\left[10^{18} \mathrm{~cm}^{-3}\right]}$. In principle, triggered injection in the plasma could achieve beam high quality (low emittance) and ultra-short durations beyond state- 
of-the-art photocathodes, due to the space-charge shielding provided by the ions in the plasma and the rapid acceleration facilitated by the ultra-high gradients.

\section{Emittance growth via plasma scattering}

Emittance growth can occur by elastic scattering of the beam and the ions in the plasma. Coulomb collisions between a beam electron and a background ion in the plasma results in a change of the rms divergence of the particle beam [11]

$$
d\left\langle\theta^{2}\right\rangle / d z=8 \pi n_{i} Z^{2} r_{e}^{2} \gamma^{-2} \ln \left(b_{\max } / b_{\min }\right)=2 k_{p}^{2} r_{e} Z \gamma^{-2} \ln \left(\lambda_{\mathrm{D}} / R_{a}\right),
$$

where $n_{i}=n_{0} / Z$ is the ion density and $Z$ is the ionization state of the ion. Here $b_{\max }=\lambda_{\mathrm{D}}$ is the plasma Debye length (screening is provided by background electrons), and $b_{\min }=R_{a}$ is the atomic radius.

Assuming linear focusing forces $\left(F_{\perp}=-k_{\beta}^{2} x_{\perp}\right)$ and an approximately matched beam, the resulting rms normalized emittance growth is $d \varepsilon_{n} / d z=\gamma k_{\beta}^{-1}\langle\theta d \theta / d z\rangle$, or

$$
\frac{d \varepsilon_{n}}{d \gamma}=\frac{k_{p}^{2} r_{e} Z \ln \left(\lambda_{\mathrm{D}} / R_{a}\right)}{\gamma k_{\beta}(d \gamma / d z)} .
$$

Equation (4) indicates that the strong focusing in a plasma-based accelerator $k_{\beta} \sim$ $k_{p} / \sqrt{\gamma}$ suppresses the emittance growth from scattering. For linear acceleration, the total emittance growth over the length of the accelerator is approximately

$$
\Delta \varepsilon_{n} \approx Z r_{e} \Phi \ln \left(\lambda_{\mathrm{D}} / R_{a}\right)\left(\gamma_{f}^{1 / 2}-\gamma_{i}^{1 / 2}\right)
$$

where $\gamma_{f}\left(\gamma_{i}\right)$ is the final (initial) beam energy, $\Phi=\left(k_{p} r_{L} / 2\right)\left(E_{z} / E_{0}\right)^{-3 / 2} / \sqrt{\cos ^{2} \Psi \sin \Psi}$, with $\Psi$ the phase of the beam in the quasi-linear wakefield, and $r_{L}$ is the transverse laser intensity gradient. Note that, for typical parameters, $\Phi \sim 1$. Equations (4) and (5) indicate that the emittance growth is only weakly dependent on plasma density. Assuming a fully-ionized Hydrogen plasma with a temperature of $T=10 \mathrm{eV}$ and a resonant laser pulse with $a_{0}=1.5$ and $r_{L}=63 \mu \mathrm{m}$, a beam injected at $\Psi=10^{\circ}$ would have an emittance growth of $\Delta \varepsilon_{n} \approx 0.4 \mathrm{~nm}$-rad after acceleration to $0.5 \mathrm{TeV}$.

There are many other sources of emittance dilution in the linac, such as misalignment between accelerating stages, vibrations, plasma fluctuations, etc. In general, the strong focusing of the plasma accelerator results in more stringent alignment tolerances due to

the small matched beam spot size $\sigma_{\perp}^{2}=\varepsilon_{n \perp} /\left(\gamma k_{\beta}\right)$. Beyond state-of-the-art beam based alignment techniques would be required to satisfy the alignment tolerances [1].

\section{Power considerations}

Operational cost of future linear colliders limit the wall plug power to a few hundred MW. In general, for efficient coupling, the bunch number will scale with plasma density 
TABLE 1. Examples of laser-plasma linear collider parameters.

\begin{tabular}{lcc}
\hline & Example 1 & Example 2 \\
\cline { 2 - 3 } Plasma number density, $n_{0}$ & $10^{17} \mathrm{~cm}^{-3}$ & $10^{18} \mathrm{~cm}^{-3}$ \\
Energy, center of mass, $E_{\mathrm{cm}}$ & $1 \mathrm{TeV}$ & $1 \mathrm{TeV}$ \\
Beam energy, $\gamma m c^{2}$ & $0.5 \mathrm{TeV}$ & $0.5 \mathrm{TeV}$ \\
Number per bunch, $N$ & $3 \times 10^{9}$ & $10^{9}$ \\
Collision rate, $f$ & $15 \mathrm{kHz}$ & $130 \mathrm{kHz}$ \\
Beam Power, $P_{b}=f N \gamma m c^{2}$ & $3.5 \mathrm{MW}$ & $10 \mathrm{MW}$ \\
Luminosity, $\mathscr{L}$ & $10^{34} \mathrm{~s}^{-1} \mathrm{~cm}^{-2}$ & $10^{34} \mathrm{~s}^{-1} \mathrm{~cm}^{-2}$ \\
Bunch length, $\sigma_{z}$ & $1 \mu \mathrm{m}$ & $1 \mu \mathrm{m}$ \\
Horizontal rms beam size at IP, $\sigma_{x}$ & $0.1 \mu \mathrm{m}$ & $0.1 \mu \mathrm{m}$ \\
Vertical rms beam size at IP, $\sigma_{y}$ & $1 \mathrm{~nm}$ & $1 \mathrm{~nm}$ \\
Horizontal normalized emittance, $\varepsilon_{n x}$ & $1 \mathrm{~mm}-\mathrm{mrad}$ & $1 \mathrm{~mm}-\mathrm{mrad}$ \\
Vertical normalized emittance, $\varepsilon_{n y}$ & $0.01 \mathrm{~mm}-\mathrm{mrad}$ & $0.01 \mathrm{~mm}-\mathrm{mrad}$ \\
Beamstrahlung parameter, $\Upsilon$ & 25 & 8.8 \\
Beamstrahlung photons per electron $n_{\gamma}$ & 0.38 & 0.17 \\
Beamstrahlung induced relative energy spread $\delta_{\gamma}$ & $10 \%$ & $4 \%$ \\
Plasma wavelength, $\lambda_{p}$ & $105 \mu \mathrm{m}$ & $33 \mu \mathrm{m}$ \\
Energy gain per stage, $W_{\text {stage }}$ & $7.4 \mathrm{GeV}$ & $0.74 \mathrm{GeV}$ \\
Single stage laser-plasma interaction length & $0.65 \mathrm{~m}$ & $2.1 \mathrm{~cm}$ \\
Drive laser coupling distance between stages & $0.5 \mathrm{~m}$ & $0.5 \mathrm{~m}$ \\
Laser energy per stage & $23 \mathrm{~J}$ & $0.8 \mathrm{~J}$ \\
Laser wavelength & $1 \mu \mathrm{m}$ & $1 \mu \mathrm{m}$ \\
Initial normalized laser intensity, $a_{0}$ & 1.5 & 1.5 \\
Average laser power per stage & $345 \mathrm{~kW}$ & $102 \mathrm{~kW}$ \\
Number of stages & 68 & 675 \\
Main linac length & $78 \mathrm{~m}$ & $0.35 \mathrm{~km}$ \\
Efficiency (wall-plug to beam) & $5 \%$ & $5 \%$ \\
Total wall-plug power & $140 \mathrm{MW}$ & $420 \mathrm{MW}$ \\
\hline
\end{tabular}

as $N \propto n^{-1 / 2}$. Therefore, for constant luminosity, and all other beam parameters fixed, the collision rate scales as $f \propto n$, and the beam power will scale as $P_{b}=f N E_{b} \propto n^{1 / 2}$. The number of stages scales as $N_{\text {stage }}=E_{b} / W_{\text {stage }} \propto n$, so the average laser power per stage scales as $P_{\text {laser }} \propto n^{-1 / 2}$ and the total wall plug power scales as $P_{\text {wall }} \propto n^{1 / 2}$.

Table 1 shows two collider examples using $n_{0}=10^{17} \mathrm{~cm}^{-3}$ or $n_{0}=10^{18} \mathrm{~cm}^{-3}$. Typical conversion efficiencies are $\sim 50 \%$ for laser to plasma wave and $\sim 30 \%$ for plasma wave to beam (shaped electron beams are assumed to avoid energy spread growth), such that the overall efficiency from laser to beam is $\sim 15 \%$. If we assume a wall-plug to laser efficiency of $\sim 33 \%$, then the efficiency from wall plug to beam is $\sim 5 \%$.

Energy deposition in a single plasma stage remaining after beam acceleration is an issue. For the $n_{0}=10^{17} \mathrm{~cm}^{-3}$ example in Table 1 , about $\sim 8 \mathrm{~J}$ of energy remains in the plasma wave after the beam exits a stage, corresponding to $\sim 120 \mathrm{~kW}$ of power. This is a significant cooling challenge. The time between bunches is $\sim 67 \mu \mathrm{s}$. This is sufficient time to allow for collisional heating of the $\left(\mathrm{Al}_{2} \mathrm{O}_{3}\right)$ capillary walls and recombination of the Hydrogen, both of which occur on the $\sim$ ns time scale. Using a H-discharge capillary for the plasma channel creation allows the H-gas to be evacuated and new gas pumped in before the arrival of the next bunch, aiding in the plasma cooling. In addition the capillary is constructed out of $\mathrm{Al}_{2} \mathrm{O}_{3}$ which has excellent heat conduction properties. 


\section{DISCUSSION AND CONCLUSIONS}

In this paper we have discussed several design considerations for future linear colliders based on laser-plasma acceleration. Based on these considerations, two examples (using $n_{0}=10^{17} \mathrm{~cm}^{-3}$ and $n_{0}=10^{18} \mathrm{~cm}^{-3}$ ) of self-consistent laser-plasma-accelerator-based collider parameters for $1 \mathrm{TeV}$ center-of-mass energy are listed in Table 1.

We have considered an electron-positron collider, but a gamma-gamma collider driven by laser plasma acceleration of electron beams can also be considered. This would also eliminate the need for positron creation and, potentially, damping rings. The scattering laser energy requirements for the gamma-gamma collider are near those required for the plasma wave excitation (e.g., tens of $\mathrm{J}$ of laser energy at the accelerator repetition rate).

Significant laser technology advances are required to realize the next-generation linear collider. Although $\sim 10 \mathrm{~J}$, short pulse lasers are currently available, repetition rates of $\sim 10 \mathrm{kHz}$ and efficiencies of $\sim 30 \%$ are presently beyond state-of-the-art laser technology. Diode-pump solid state lasers show promise to generate hundreds of $\mathrm{kW}$ with high efficiency in the next decade. In addition there is significant laser-plasma accelerator $\mathrm{R} \& \mathrm{D}$ required before realization of a laser-plasma-accelerator-based linear collider is possible. In particular, these include demonstration of accelerator stage coupling, detailed control of beam injection, and maintaining high beam quality over the length of the accelerator. A TeV linear collider is extremely challenging for any technology, but laser-plasma-based accelerators continue to show great promise as a solution to address the size of future linear colliders.

\section{ACKNOWLEDGMENTS}

This work was supported by the Director, Office of Science, Office of High Energy Physics, of the U.S. Department of Energy under Contract No. DE-AC02-05CH11231.

\section{REFERENCES}

1. G. Dugan, "Advanced Accelerator System Requirements for Future Linear Colliders," in Advanced Accelerator Concepts, edited by V. Yakimenko, AIP, 2004, vol. 737, pp. 29-60.

2. E. Esarey, C. B. Schroeder, and W. P. Leemans, Rev. Mod. Phys. (2009, in press).

3. W. P. Leemans, B. Nagler, A. J. Gonsalves, C. Tóth, K. Nakamura, C. G. R. Geddes, E. Esarey, C. B. Schroeder, and S. M. Hooker, Nature Phys. 2, 696-699 (2006).

4. K. Nakamura, B. Nagler, C. Tóth, C. G. R. Geddes, C. B. Schroeder, E. Esarey, W. P. Leemans, A. J. Gonsalves, and S. M. Hooker, Phys. Plasmas 14, 056708 (2007).

5. C. G. R. Geddes, C. Toth, J. van Tilborg, E. Esarey, C. B. Schroeder, J. Cary, and W. P. Leemans, Phys. Rev. Lett. 95, 145002 (2005).

6. P. Sprangle, B. Hafizi, J. R. Peñano, R. F. Hubbard, A. Ting, C. I. Moore, D. F. Gordon, A. Zigler, D. Kaganovich, and T. M. Antonsen, Jr., Phys. Rev. E 63, 056405 (2001).

7. B. A. Shadwick, G. M. Tarkenton, E. H. Esarey, and W. P. Leemans, IEEE Trans. Plasma Sci. 30, 38 (2002).

8. D. Panasenko et al. (2008), in these Proceedings.

9. K. Oide, Phys. Rev. Lett. 61, 1713-1715 (1988).

10. A. W. Chao, and M. Tigner, editors, Handbook of accelerator physics and engineering, World Scientific, Singapore, 1999.

11. D. R. Nicholson, Introduction to Plasma Theory, Krieger, 1992. 